\title{
Fast Groupwise 4D Deformable Image Registration for Irregular Breathing Motion Estimation
}

\author{
Bartłomiej W. Papież $\dot{z}^{1,2}$, Daniel R. McGowan ${ }^{3,4}$, Michael Skwarski ${ }^{3}$, \\ Geoff S. Higgins ${ }^{3}$, Julia A. Schnabel ${ }^{2,5}$, and Sir Michael Brady ${ }^{3}$ \\ 1 Big Data Institute, Li Ka Shing Centre for Health Information and Discovery, \\ University of Oxford, UK \\ 2 Institute of Biomedical Engineering, \\ Department of Engineering Science, University of Oxford, Oxford, UK \\ 3 Department of Oncology, University of Oxford, Oxford, UK \\ ${ }^{4}$ Radiation Physics and Protection, Oxford University Hospitals NHS FT, Oxford, \\ UK \\ 5 Department of Biomedical Engineering, School of Biomedical Engineering and \\ Imaging Sciences, King's College London, London, UK
}

\begin{abstract}
Tumor heterogeneity can be assessed quantitatively by analyzing dynamic contrast-enhanced imaging modalities potentially leading to improvement in the diagnosis and treatment of cancer, for example of the lung. However, the acquisition of standard lung sequences is often compromised by irregular breathing motion artefacts, resulting in unsystematic errors when estimating tissue perfusion parameters. In this work, we illustrate implicit deformable image registration that integrates the Demons algorithm using the local correlation coefficient as a similarity measure, and locally adaptive regularization that enables incorporation of both spatial sliding motions and irregular temporal motion patterns. We also propose a practical numerical approximation of the regularization model to improve both computational time and registration accuracy, which are important when analyzing long clinical sequences. Our quantitative analysis of $4 \mathrm{D}$ lung Computed Tomography and Computed Tomography Perfusion scans from clinical lung trial shows significant improvement over state-of-the-art pairwise registration approaches.
\end{abstract}

\section{Introduction}

Dynamic imaging modalities such as Computed Tomography Perfusion (CTP) or Dynamic Contrast Enhanced Magnetic Resonance Imaging (DCE-MRI) have attracted significant interest in quantitative oncological imaging since they have the great potential for the assessment of tumor heterogeneity, leading, in turn, to improvements in diagnosis and the formulation of personalized patient treatment plan $[5,10]$. These dynamic modalities have been widely used in clinical applications related to brain [7] or head and neck radiotherapy [4], but their application has been rather limited in lung and abdominal radiotherapy, not least because 
of the deformations caused primarily by breathing [20]. The complexity of human lung motion and the irregular temporal motion patterns rule out generic deformable image registration methods as they are unable to model accurately the properties of the relevant tissues. Therefore, modeling and analyzing lung and abdominal motion has been recognized as an important element of many biomedical image analysis applications [16].

\subsection{Related work}

The standard approach to motion correction of dynamic sequences is to perform state-of-the-art pairwise deformable registration algorithms [17] between the reference and the follow-up volumes from a sequence. While such an approach is straightforward, it results in inverse inconsistency and transitivity errors [6] of the estimated transformations due to accumulation of errors in the sequence. Such errors are then propagated to any subsequent pharmacokinetic analysis. Additionally, there is bias introduced in the registration results due to the selection of a fixed reference volume, since choosing an outlying reference volume may be disadvantageous as all registrations will need to estimate inadequate large displacement fields. To reduce errors related to estimation of the displacement fields, numerous methods have incorporated temporal smoothness constraints $[12,1,2,18]$. However, temporal regularization models do not solve the problem of the fixed reference volume, and so temporal groupwise image registration has been proposed $[22,13,21]$. Simultaneous registration of all images in a sequence reduces the bias introduced by a fixed reference volume, while temporal regularization preserves smoothness of the estimated displacement fields. Although such an approach is more robust to outliers, temporal smoothness is implausible for irregular motion artefacts, which are apparent for patients with lung cancer or other co-morbidities.

Contributions. We explore groupwise deformable image registration [22] to dynamic lung Computed Tomography (CT) as a method that is intrinsically invariant to the selection of reference volume and irregularity of lung expiration/inspiration motion pattern. In particular, we developed a groupwise deformable image registration derived from the LCC Demons [11] with adaptive regularization using local spatial and temporal filtering of the estimated displacement fields [14]. The main contributions of the manuscript are as follows: we extend 3D guided image filtering to its $4 \mathrm{D}$ counterpart to enable efficient spatiotemporal regularization of the estimated displacement fields from groupwise image registration. Guided image filtering [9] is a computationally attractive, linear image filtering technique, and here we used it to propagate spatio-temporal information from a so-called guidance image to the regularization. We present a numerical approximation which significantly reduces the computational burden whilst improving registration accuracy, which is important when dealing with large 4D data set. The improved performance on a publicly available lung CT data set [3] is quantitatively assessed. Finally, the robustness of the method on 
a challenging clinical application of CTP motion compensation for patients with non-small cell lung cancer is demonstrated.

\section{Methods}

\subsection{Classic Groupwise Deformable Image Registration}

In the classic formulation [6], groupwise deformable image registration is defined as a global energy minimization problem:

$$
\hat{\mathbf{u}}=\arg \min _{\boldsymbol{u}}(\varepsilon(\boldsymbol{u})=\operatorname{Sim}(\mathbf{I}(\boldsymbol{u}))+\alpha \operatorname{Reg}(\boldsymbol{u}))
$$

with respect to a set of displacement fields $\boldsymbol{u}$ describing geometrical correspondences between a set of $M$ input images $\mathbf{I}=\left[I_{m}: \Omega \rightarrow \mathbb{R}, \Omega \in \mathbb{R}^{3}, m=1, \ldots M\right]$. If a reference image $I_{r e f}$ for groupwise deformable image registration is explicitly provided, the process of estimation of the set of displacement fields to such a reference image is called reference-based groupwise registration and the objective function $\varepsilon$ is defined as follows:

$$
\varepsilon(\boldsymbol{u})=\sum_{m=1}^{M} \operatorname{Sim}\left(I_{r e f}, I_{m}\left(\boldsymbol{u}_{m}\right)\right)+\alpha \sum_{m=1}^{M} \operatorname{Reg}\left(\boldsymbol{u}_{m}\right)
$$

In the case when the reference image $I_{r e f}$ is not provided, the estimation of the set of the displacement fields from each image $I_{m}$ in the set $\mathbf{I}$ is performed with respect to the unknown reference image, considering minimization the sum of the similarity measure Sim between each pair of the input images as follows:

$$
\varepsilon(\boldsymbol{u})=\sum_{m=1}^{M} \sum_{\substack{n=1 \\ n \neq m}}^{M} \operatorname{Sim}\left(I_{m}\left(\boldsymbol{u}_{m}\right), I_{n}\left(\boldsymbol{u}_{n}\right)\right)+\alpha \sum_{m=1}^{M} \operatorname{Reg}\left(\boldsymbol{u}_{m}\right)
$$

The objective function of the implicit groupwise deformable image registration defined by Eq. (3) can be solved using a variety of methods. Because of its simplicity and efficiency, we choose the Demons framework [19] to solve Eq. (3). For the Demons framework, the optimization procedure alternates between minimizing the energy related to the similarity measure Sim and the regularization Reg in an iterative manner. The contributions of this work will be described in detail in the following sections.

\subsection{Groupwise similarity measure}

From the fact that the symmetric image registration can be seen as an implicit groupwise image registration with only two input images, the minimization process of groupwise registration (Eq. (3)) is similar to the symmetric registration [11]. In the standard Demons registration, the displacement field is estimated by the minimization of the sum of the squared differences (SSD) between the input 
images. In the case of the time scans acquired with contrast agent, the SSD is not viable choice since it is not robust to the local intensity changes caused by wash-in and wash-out of contrast. Here, we propose to replace SSD-based groupwise registration to the local correlation coefficient (LCC) based counterpart. LCC-Demons has already been used for symmetric brain MRI registration due to the independence of any additive and multiplicative noise in the data [11]. The LCC similarity measure between a pair of images $I_{1}$ and $I 2$ defined for symmetric registration is defined in the following way:

$$
L C C\left(I_{1}\left(\boldsymbol{u}_{1}\right), I_{2}\left(\boldsymbol{u}_{2}\right)\right)=\frac{\bar{I}_{1}\left(\boldsymbol{u}_{1}\right) \bar{I}_{2}\left(\boldsymbol{u}_{2}\right)}{\left(\bar{I}_{1}\left(\boldsymbol{u}_{1}\right)\right)^{2}\left(\bar{I}_{2}\left(\boldsymbol{u}_{2}\right)\right)^{2}}
$$

where $\bar{I}_{1}$ and $\bar{I}_{2}$ are the local mean image intensities for image $I_{1}$ and $I_{2}$, respectively. Following the derivation in [11], the LCC symmetric update of the displacement field for the Demons can be calculated with a closed form formula as follows:

$$
\boldsymbol{d u}_{12}(\boldsymbol{x})=\frac{-2 \text { force }_{l c c}}{\left(\text { force }_{l c c}+\sigma_{\text {noise }}^{2}\right)}
$$

at any spatial position $\boldsymbol{x}$ in the image domain, $\sigma_{\text {noise }}^{2}$ is a noise estimator, and

$$
\text { force }_{l c c}=G *\left(\frac{I_{1} \nabla I_{2}^{T}}{I_{1} I_{2}}-\frac{I_{2} \nabla I_{1}^{T}}{I_{1} I_{2}}+\frac{I_{1} \nabla I_{1}^{T}}{I_{1}^{2}}-\frac{I_{2} \nabla I_{2}^{T}}{I_{2}^{2}}\right)
$$

where $G *$ is a Gaussian kernel for smoothing. Finally, the average update of the displacement field for the groupwise registration is calculated using the logEuclidean mean for vector field $\boldsymbol{d u}(\boldsymbol{x})$ given by:

$$
\boldsymbol{d} \boldsymbol{u}_{m}(\boldsymbol{x})=\frac{1}{M-1} \sum_{\substack{n=1 \\ n \neq m}}^{M}\left(\boldsymbol{d} \boldsymbol{u}_{m n}(\boldsymbol{x})\right)
$$

\subsection{Spatio-temporal filtering of displacement fields}

Motion correction for intra-subject dynamic imaging of lungs is challenging due to the complexity of motion to be estimated stemming from patient breathing during acquisition. The standard regularization model of the Demons framework realized by Gaussian smoothing of the estimated displacement field has been shown to be inadequate to model respiratory motion. Here, we extend a previous approach [14], where spatially adaptive filtering of displacement field using the guided image filtering technique was developed, and we present a generic approach for $4 \mathrm{D}$ regularization. In our approach, the estimated displacement field is additionally filtered by considering the temporal context of the guidance information encoded in the dynamic imaging.

Following $[15,14]$, the initial displacement field $\boldsymbol{u}_{i n}$ is first spatially filtered considering the context of the guidance information $I_{g}$ as follows:

$$
\boldsymbol{u}_{t m p}(\boldsymbol{x})=\sum_{\boldsymbol{y} \in \mathcal{N}} W_{\text {spatial }}\left(I_{g}, \boldsymbol{x}, \boldsymbol{y}\right)\left(\boldsymbol{u}_{\text {in }}(\boldsymbol{y})+\boldsymbol{d} \boldsymbol{u}_{m}(\boldsymbol{y})\right)
$$


where $\mathcal{N}$ is the local spatial neighborhood (a box window of size $r_{\mathcal{N}}$ ), and $W_{\text {spatial }}$ are the kernel weights for spatial filter. Similarly, the filtered displacement field $\boldsymbol{u}_{t m p}$ is then temporally filtered again using the dynamic context of the guidance information $I_{g}(t)$ in the following way:

$$
\boldsymbol{u}_{\text {out }}(\boldsymbol{x})=\sum_{t \in \mathcal{T}} W_{\text {temporal }}\left(I_{g}, \boldsymbol{x}, t\right)\left(\boldsymbol{u}_{\text {tmp }}(\boldsymbol{x}, t)+\boldsymbol{d} \boldsymbol{u}_{m}(\boldsymbol{x}, t)\right)
$$

where $\mathcal{T}$ is the local temporal neighborhood (a box window of size $r_{\mathcal{T}}$ ), and $W_{\text {temporal }}$ are the kernel weights for temporal filter.

Our regularization method is composed of two main steps: spatial adaptive filtering of the displacement field to enforce discontinuity preserving properties at lung interfaces; and adaptive temporal filtering of the displacement field to ensure capture of temporal (ir)regularities of the patient's motion pattern. In our framework, these two steps could not be combined in one step of filtering the estimated displacement field, because the local spatial neighborhood and the local temporal neighborhood have different units, and respective parameters $r_{\mathcal{N}}$ and $r_{\mathcal{T}}$ need to be setup separately.

Regularization with subsampled guided image filter Since medical volumes can easily be $512 \times 512 \times 120$ or larger, a linear time (with respect of the number of voxels) can be still considered to be computationally significant, particularly since the filtering procedure is repeated at every iteration of the presented deformable registration. Therefore, we adapt a speed-up strategy for fast guided filters mentioned in [8] to improve performance of the presented filteringbased regularization. Most of the computation time for the guided filters is spent on the estimation of the filter coefficients, however the coefficients do not need to be estimated from full-resolution volumes. Therefore, to estimate those coefficients, we subsample the input and guidance image by factor $s$, and perform all computations related to filtering of the displacement on the subsampled volumes. Then, the coefficients estimated from the subsampled volumes are upsampled to the original size of the volumes, and then the final step of guided filtering is performed using the original guidance image $I_{G}$ and the upsampled coefficients. Due to the use of a subsampled image to estimate the filter coefficients, the computational cost of filtering can be reduced approximately by $s^{2}$ as only the final step is performed on full size volume. Comparison of the influence of filtering of the estimated displacement using the subsampled volumes is shown in Sec. 3.2.

\section{Experiments}

\subsection{Data description}

For quantitative evaluation of the proposed regularization method we use a publicly available 4D CT data set [3]. The Dir-Lab data set consists of 10 consecutive respiratory cycle phase volumes with spatial resolution varying between 
Table 1. Results achieved by the proposed method with different value of the subsampling factor $s$ for $4 \mathrm{D}$ registration of CT lung from Dir-Lab data set. The method with the subsampling factor $s=4$ shows the lowest average Target Registration Error (TRE) among all methods.

\begin{tabular}{cclllllll}
\hline & & \multicolumn{7}{c}{ subsampling factor $s$} \\
& before & $s=1[14]$ & \multicolumn{1}{c}{$s=2$} & \multicolumn{1}{c}{$s=3$} & \multicolumn{1}{c}{$s=4$} & \multicolumn{1}{c}{$s=5$} & \multicolumn{1}{c}{$s=6$} \\
\hline$c 1$ & $3.89 \pm 2.9$ & $0.95 \pm 0.9$ & $1.08 \pm 1.1$ & $0.88 \pm 1.0$ & $0.90 \pm 1.0$ & $\mathbf{0 . 8 6} \pm \mathbf{1 . 0}$ & $\mathbf{0 . 8 6} \pm \mathbf{1 . 0}$ \\
c2 & $4.34 \pm 3.9$ & $0.95 \pm 1.0$ & $1.00 \pm 1.0$ & $0.98 \pm 1.1$ & $0.94 \pm 1.0$ & $\mathbf{0 . 9 1} \pm \mathbf{1 . 0}$ & $0.92 \pm 1.0$ \\
$c 3$ & $6.94 \pm 4.1$ & $1.06 \pm 1.1$ & $1.09 \pm 1.1$ & $\mathbf{1 . 0 3} \pm \mathbf{1 . 1}$ & $1.06 \pm 1.1$ & $1.08 \pm 1.1$ & $1.27 \pm 1.2$ \\
$c 4$ & $9.83 \pm 4.9$ & $3.19 \pm 4.8$ & $3.16 \pm 4.3$ & $2.71 \pm 3.7$ & $\mathbf{2 . 5 3} \pm \mathbf{3 . 2}$ & $2.62 \pm 3.1$ & $2.70 \pm 3.0$ \\
$c 5$ & $7.48 \pm 5.5$ & $1.40 \pm 1.5$ & $1.44 \pm 1.6$ & $1.33 \pm 1.5$ & $\mathbf{1 . 3 1} \pm \mathbf{1 . 5}$ & $1.41 \pm 1.5$ & $1.51 \pm 1.5$ \\
$c 6$ & $10.9 \pm 7.0$ & $2.74 \pm 3.2$ & $2.63 \pm 3.2$ & $2.19 \pm 2.5$ & $1.89 \pm 1.9$ & $\mathbf{1 . 8 2} \pm \mathbf{1 . 6}$ & $1.88 \pm 1.4$ \\
$c 7$ & $11.0 \pm 7.4$ & $1.69 \pm 1.6$ & $1.67 \pm 1.6$ & $\mathbf{1 . 5 2} \pm \mathbf{1 . 4}$ & $\mathbf{1 . 5 2} \pm \mathbf{1 . 4}$ & $1.74 \pm 1.6$ & $1.91 \pm 1.4$ \\
$c 8$ & $15.0 \pm 9.0$ & $2.45 \pm 3.1$ & $2.10 \pm 2.6$ & $\mathbf{1 . 8 5} \pm \mathbf{2 . 3}$ & $1.87 \pm 2.3$ & $2.02 \pm 2.4$ & $2.35 \pm 2.4$ \\
$c 9$ & $7.92 \pm 4.0$ & $1.56 \pm 1.0$ & $\mathbf{1 . 3 1} \pm \mathbf{1 . 2}$ & $1.33 \pm 1.1$ & $1.37 \pm 1.1$ & $1.55 \pm 1.2$ & $1.79 \pm 1.2$ \\
$c 10$ & $7.30 \pm 6.4$ & $1.46 \pm 1.8$ & $1.40 \pm 1.7$ & $1.36 \pm 1.5$ & $\mathbf{1 . 2 7} \pm \mathbf{1 . 4}$ & $1.29 \pm 1.3$ & $1.43 \pm 1.3$ \\
\hline$T \bar{R} E$ & $8.46 \pm 5.5$ & $1.71 \pm 0.8$ & $1.69 \pm 0.7$ & $1.52 \pm 0.6$ & $\mathbf{1 . 4 7} \pm \mathbf{0 . 5}$ & $1.53 \pm 0.5$ & $1.66 \pm 0.6$
\end{tabular}

$0.97 \times 0.97 \times 2.5$ and $1.16 \times 1.16 \times 2.5 \mathrm{~mm}^{3}$. To quantify registration accuracy, the Target Registration Error (TRE) was calculated for the well-distributed set of landmarks, which are provided with this data set (300 landmarks per case for inhale and exhale volumes).

For the second experiment, the method was evaluated on CTP data from a clinical trial (NCT02628080) acquired at the Churchill Hospital in Oxford evaluating whether an investigational drug (atovaquone) alters tumour hypoxia. The data reported here are for the pre-atovaquone scans. For the CTP patients are imaged supine on a GE Discovery 710 PET/CT scanner with a 45s cine mode CT using $120 \mathrm{kV}$ and $60 \mathrm{~mA}$. During this $70 \mathrm{~mL}$ contrast (Omnipaque 300) is injected at $5 \mathrm{~mL} / \mathrm{s}$ followed by $25 \mathrm{~mL}$ water at $5 \mathrm{~mL} / \mathrm{s}$. The patient is instructed to hold their breath for as long as possible (at inspiration) and if necessary to breathe out very slowly.

\subsection{Results for publicly available $4 \mathrm{D}$ lung CT dataset}

Table 1 shows the TRE based on 300 well-populated, manually annotated landmarks for all ten cases included in the Dir-Lab data set [3]. The initial TRE is $8.46 \pm 5.5 \mathrm{~mm}$ and the transformations estimated by the proposed method with subsampling factor $s=4$ reduces the TRE to $1.47 \pm 0.5 \mathrm{~mm}$, achieving the best result in our comparison.

Visualization of the results for the presented method is shown in Fig. 1. Red arrows depict regions of interest where the presented method with the subsampling factor $s=4$ outperformed the baseline method with the subsampling factor $s=1$. 

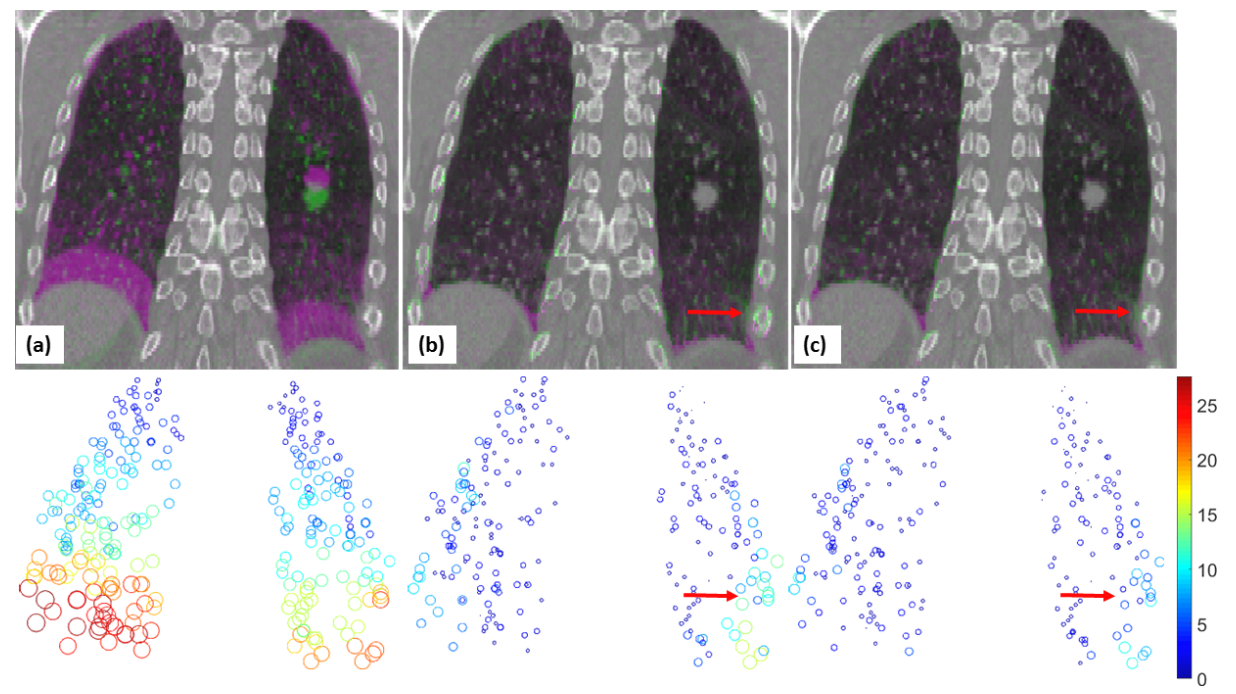

Fig. 1. Visualization of the image intensity differences (top) and 2D projection of the Target Registration Error (bottom) before registration (a), and after performing the proposed method with the subsampling factor (b) $s=1$ [14], and (c) $s=4$ for the challenging case 6 from Dir-Lab. Color overlay is given for the coronal view of inhale (green) and exhale (magenta) volumes. TRE is projected on the coronal plane and denoted by the size and color of circles. A clear improvement after registration using the presented method is visible (labeled by red arrows).

\subsection{Results for lung tumor CTP}

At the time of writing, we have analyzed 11 dynamic 4D-CTP scans of patients who have a lung tumor. Registration quality was evaluated via the Correlation Coefficient (CC), and for all data sets, and noticeable improvement was found (avg. CC before $=0.96$, and after $=0.99$ ). Fig 2 shows an axial view of an exemplar CTP and the time-cuts, which demonstrate visual improvement in alignment over acquisition time of CTP volumes.

\section{Discussion and Conclusions}

In this paper, we have presented a new class of $4 \mathrm{D}$ regularization model based on $4 \mathrm{D}$ guided image filtering, that can be easily incorporated into groupwise deformable image registration. Furthermore, we have shown that the use of the subsampled guided image to calculate the filter's coefficient for the displacement fields improves the registration accuracy while reduces computational cost of registration. This is particularly important for long temporal acquisitions such as DCE-MRI or CTP, which consists of several volumes. From a clinical perspective, our registration framework compensates for misalignment between consecutive CTP volumes caused by patient-specific breath-hold variability, resulting in the 

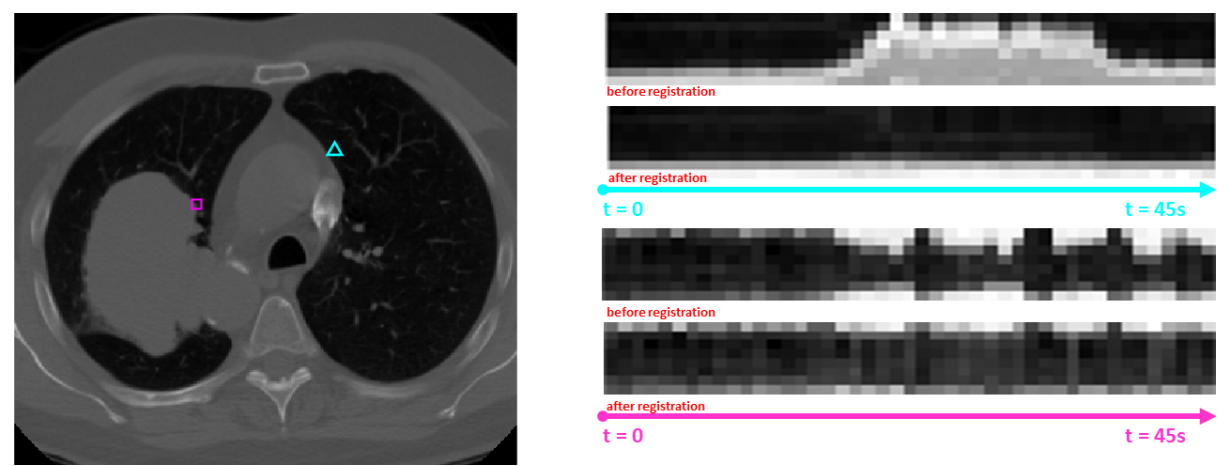

Fig. 2. Axial (left) view for reference volume with cyan and magenta pointers indicating the locations of their corresponding time-cuts for CTP in the challenging case \#AT010 before and after registration using the proposed method with the subsampling factor (b) $s=4$.

improved alignment of structure of interest in the lungs. We obtained a good visual alignment of the CTP data, however actual registration errors measured by the densely distributed landmarks was not assessed. Manual annotations of temporal functional imaging e.g. Computed Tomography Perfusion is inevitably labor-intensive. Furthermore, intensity changes caused by contrast uptake or the low contrast of lung tissue in Computed Tomography Perfusion makes accurate annotation even more challenging. For these reasons, we compared our method using publicly available lung 4D CT data set [3]. We next aim to quantify the impact of our method on estimation of tissue perfusion parameters.

Acknowledgments. We acknowledge funding from the CRUK/ EPSRC Cancer Imaging Centre in Oxford. The ATOM trial is sponsored by the University of Oxford and coordinated by the Oncology Clinical Trials Office. It is supported by the Howat Foundation, Oxford Cancer Imaging Centre, Cancer Research UK, National Institute of Health Research, Oxford Biomedical Research Centre and the ECMC. BWP acknowledges Oxford NIHR Biomedical Research Centre (Rutherford Fund).

\section{References}

1. W. Bai and M. Brady. Regularized B-spline deformable registration for respiratory motion correction in PET images. Phys Med Biol, 54(9):2719, 2009.

2. E. Castillo, R. Castillo, J. Martinez, M. Shenoy, and T. Guerrero. Four-dimensional deformable image registration using trajectory modeling. Phys Med Biol, 55(1):305, 2009.

3. R. Castillo, E. Castillo, R. Guerra, V. Johnson, T. McPhail, A. Garg, and T. Guerrero. A framework for evaluation of deformable image registration spatial accuracy using large landmark point sets. Phys. Med. Biol., 54:1849-1870, 2009. 
4. O.I. Craciunescu, D.S. Yoo, E. Cleland, N. Muradyan, M.D. Carroll, J.R. MacFall, D.P. Barboriak, and D.M. Brizel. Dynamic contrast-enhanced MRI in head-andneck cancer: the impact of region of interest selection on the intra-and interpatient variability of pharmacokinetic parameters. International Journal of Radiation Oncology* Biology* Physics, 82(3):e345-e350, 2012.

5. R. García-Figueiras, V.J. Goh, A.R. Padhani, S. Baleato-González, M. Garrido, L. León, and A. Gómez-Caamaño. CT perfusion in oncologic imaging: a useful tool? American Journal of Roentgenology, 200(1):8-19, 2013.

6. X. Geng, G.E Christensen, H. Gu, T.J Ross, and Y. Yang. Implicit reference-based group-wise image registration and its application to structural and functional MRI. Neuroimage, 47(4):1341-1351, 2009.

7. F. Godenschweger, U. Kägebein, D. Stucht, U. Yarach, A Sciarra, R Yakupov, F Lüsebrink, P Schulze, and O Speck. Motion correction in MRI of the brain. Phys Med Biol, 61(5):R32, 2016.

8. K. He and J. Sun. Fast guided filter. arXiv preprint arXiv:1505.00996, 2015.

9. K. He, J. Sun, and X. Tang. Guided image filtering. IEEE Trans. Pattern Anal. Mach. Intell., 35(6):1397-1409, 2013.

10. H. Koyama, Y. Ohno, S. Seki, M. Nishio, T. Yoshikawa, S. Matsumoto, and K. Sugimura. Magnetic resonance imaging for lung cancer. Journal of thoracic imaging, 28(3):138-150, 2013.

11. M. Lorenzi, N. Ayache, G. B. Frisoni, X. Pennec, and Alzheimer's Disease Neuroimaging Initiative (A. D. N. I) . LCC-Demons: a robust and accurate symmetric diffeomorphic registration algorithm. Neuroimage, 81:470-483, Nov 2013.

12. J.R. McClelland, J.M. Blackall, S. Tarte, A.C. Chandler, S. Hughes, S. Ahmad, D.B. Landau, and D.J. Hawkes. A continuous 4D motion model from multiple respiratory cycles for use in lung radiotherapy. Med. Phys., 33(9):3348-3358, 2006.

13. CT Metz, S. Klein, M. Schaap, T. van Walsum, and WJ Niessen. Nonrigid registration of dynamic medical imaging data using $\mathrm{nD}+\mathrm{t}$ B-splines and a groupwise optimization approach. Med Image Anal, 15(2):238-249, 2011.

14. B.W. Papież, J. Franklin, M.P. Heinrich, F.V. Gleeson, and J.A. Schnabel. Liver motion estimation via locally adaptive over-segmentation regularization. In Medical Image Computing and Computer-Assisted InterventionMICCAI 2015, pages 427434. Springer, 2015.

15. B.W Papież, M.P Heinrich, J. Fehrenbach, L. Risser, and J.A Schnabel. An implicit sliding-motion preserving regularisation via bilateral filtering for deformable image registration. Med. Image Anal., 18(8):1299-1311, 2014.

16. J.A Schnabel, M.P. Heinrich, B.W. Papież, and J.M. Brady. Advances and challenges in deformable image registration: From image fusion to complex motion modelling. Medical Image Analysis, 33:145-148, 2016.

17. A. Sotiras, C. Davatzikos, and N. Paragios. Deformable medical image registration: A survey. IEEE Trans. Med. Imaging, 32(7):1153-1190, 2013.

18. J. Vandemeulebroucke, S. Rit, J. Kybic, P. Clarysse, and D. Sarrut. Spatiotemporal motion estimation for respiratory-correlated imaging of the lungs. Med. Phys., 38(1):166-178, Jan 2011.

19. T. Vercauteren, X. Pennec, A. Perchant, and N. Ayache. Diffeomorphic Demons: Efficient non-parametric image registration. Neuroimage, 45:S61-S72, 2009.

20. M. von Siebenthal, G. Szekely, U. Gamper, P. Boesiger, A. Lomax, and P. Cattin. $4 \mathrm{D}$ MR imaging of respiratory organ motion and its variability. Phys Med Biol, 52(6):1547, 2007. 
21. G. Wu, Q. Wang, D. Shen, Alzheimer's Disease NeuroImaging Initiative, et al. Registration of longitudinal brain image sequences with implicit template and spatialtemporal heuristics. NeuroImage, 59(1):404-421, 2012.

22. M. Yigitsoy, C. Wachinger, and N. Navab. Temporal groupwise registration for motion modeling. In Information Processing in Medical Imaging, pages 648-659. Springer, 2011. 